\title{
REGISTADORES AUTOMÁTICOS DE BAIXO-CUSTO PARA A MONITORIZAÇÃO DE TEMPERATURAS DO AR, DA ROCHA E DO SOLO
}

\author{
Gonçalo Teles Vieira ${ }^{1}$ \\ CARLA MORA ${ }^{2}$ \\ Miguel RAMOS ${ }^{3}$
}

\begin{abstract}
Resumo - Registadores automáticos de temperatura do tipo Tiny Talk II ${ }^{\circledR}$ foram transformados no laboratório do Centro de Estudos Geográficos, com o objectivo de monitorizar as variações de temperatura do ar, rocha e solo. Tratam-se de registadores de 8 bits, com capacidade para armazenar até 1800 dados de temperatura medida a intervalos pré-definidos. A principal vantagem do sistema reside no baixo custo dos instrumentos, na sua pequena dimensão e na simplicidade das adaptações necessárias para que sejam medidos os parâmetros referidos. Descrevem-se na presente nota todos os procedimentos necessários para a construção dos três tipos de sistema de aquisição de dados.
\end{abstract}

Palavras-chave: Registador automático, temperatura, clima local, periglaciar

Abstract - LOW-COST DATA LOGGERS FOR AIR, SOIL AND ROCK TEMPERATURE MONITORING - Tiny Talk II data loggers were adapted in the laboratory of the Centro de Estudos Geográficos for air, soil and rock temperature monitoring. The loggers are single-channel 8 bit devices with a $2 \mathrm{~K}$ EEPROM memory that allows the registration of 1,800 temperature records at user-defined intervals. Temperatures are measured by NTC100 thermistors separated by the authors from the data logger for more accurate measurements. Data is transferred from the loggers into a portable computer using a RS-232 cable. It is a low cost system very appropriate for use in rough environmental conditions, particularly in mountain areas. It was successfully tested by the authors in the Antarctic, Pyrenees, Sierra Nevada and Serra da Estrela.

The detailed procedures for the construction of three measuring systems are described. The air temperature apparatus is constructed using PVC tubing and protecting cases and is designed for the measurement of air temperatures at $130 \mathrm{~cm}$ above the ground. The soil temperature monitoring apparatus measures temperatures at 1, 5, 10 and $20 \mathrm{~cm}$ depth and is based in 4 data loggers protected from moisture and dust in an IP68 case. The rock temperature measuring system is similar to the latter but the sensors are installed in the interior of the rock and isolated using silicon glue.

Key words: data logger, temperature, local climate, periglacial.

1 Investigador do Centro de Estudos Geográficos. Assistente da Universidade de Lisboa (Endereço do CEG no início do volume). E-mail: gtvieira@ceg.ul.pt

2 Investigadora do Centro de Estudos Geográficos, Universidade de Lisboa (Endereço do CEG no início do volume). E-mail: cmora@ceg.ul.pt

3 Departamento de Física, Universidade de Alcalá de Henares, 28871 Alcalá de Henares, Espanha, Tel: + 34 918854917, Fax: + 34 918854942. E-mail: fsmrs@alcala.es 


\section{INTRODUÇÃO}

A monitorização do regime térmico do ar, com intervalos temporais curtos e usando uma rede de estações densa, é um aspecto importante em estudos de climatologia local, permitindo conhecer a variação espacial e temporal da temperatura. Estes dados podem ser posteriormente usados na construção de modelos que, elaborados para condições meteorológicas previamente escolhidas, ou para as condições médias, podem ser usados como ferramenta no ordenamento do território. Exemplo disso, é o mapa de risco de geada e de nevoeiro de irradiação apresentado por ALCOFORADO et al. (1993) para o vale da ribeira da Ajuda, na serra da Arrábida.

Em geomorfologia, para se compreender a influência do regime térmico da rocha ou do solo, nos processos que os afectam, é necessário proceder à monitorização da temperatura a várias profundidades. Pode-se assim conhecer a migração das frentes de frio ou de calor na rocha e no solo, e estudar as suas diferentes reacções (por exemplo, a crioclastia, a termoclastia ou a crioexpulsão).

A monitorização sincrónica das temperaturas do ar e do substrato tem, ainda, o acrescido interesse de permitir avaliar as relações entre ambas, e aferir a validade da utilização da primeira, para estimar a segunda, aspecto corrente em estudos de geomorfologia periglaciária, em áreas marcadas pela escassez de dados (ver por exemplo THORN et al., 1999).

O objectivo principal do projecto ESTRELA (Praxis/C/CTE/11153/1998) é o estudo da paisagem da serra da Estrela, e das relações entre a dinâmica geomorfológica, climática e fitossociológica a diferentes escalas espaciais. Numa área de montanha como a que estudamos, com uma altitude que atinge 1993 $\mathrm{m}$, um dos factores mais importantes para as comunidades vegetais e para a dinâmica geomorfológica é a temperatura, e o seu comportamento no solo, na rocha e no ar. A topografia movimentada da serra gera uma influência muito significativa nas condições térmicas do ar e do solo, pelo que, para conhecer o seu comportamento na área de trabalho, se tornou necessária a instalação de uma rede de estações relativamente densa. As estações foram colocadas em posições topográficas e em tipos de substrato escolhidos, de modo a melhor poder modelizar a diversidade das condições da serra.

Mas a aquisição e instalação de instrumentação automática é onerosa, o que inviabiliza muitos estudos que se fazem a nível universitário, meio em que o orçamento é, geralmente, o principal condicionante da investigação. A solução que encontrámos foi adquirir pequenos registadores de dados automáticos e, no laboratório do Centro de Estudos Geográficos, modificar as suas características, adaptando-os para os objectivos que nos propúnhamos alcançar. Foi deste modo possível construir uma rede de estações de monitorização de temperaturas de baixo custo, que nos permitirá aprofundar o conhecimento, até agora praticamente inexistente, das caraterísticas da temperatura do ar e do substrato da mais alta montanha de Portugal Continental. Esta abordagem 
foi possível, graças à colaboração que se vem desenvolvendo entre investigadores do Centro de Estudos Geográficos da Universidade de Lisboa e do Grupo de Investigação de Física Ambiental da Universidade de Alcalá de Henares. A presente nota aborda a concepção e características da instrumentação desenvolvida.

\section{A INSTRUMENTAÇÃO PARA MONITORIZAÇÃO DE TEMPERATURAS}

\section{Especificações técnicas gerais}

A instrumentação desenvolvida baseia-se no sistema de aquisição automática e registo de dados de 1 canal Tiny Talk II ${ }^{\circledR}$ da Gemini Data Loggers UK Ltd., disponíveis comercialmente a um custo relativamente baixo. Existem no mercado vários tipos de data logger de características semelhantes, produzidos por diferentes firmas, e que facilmente poderão ser adaptados para os mesmos fins.

A escolha do sistema Tiny Talk II@ prende-se com a sua pequena dimensão (diâmetro $=34 \mathrm{~mm}$, comprimento $=52 \mathrm{~mm}$ ), robustez, facilidade de aquisição em Portugal, e com os bons resultados com ele obtidos noutras áreas de trabalho, sob condições meteorológicas particularmente rigorosas, como os Pirenéus, Serra Nevada e Antárctida (RAmos, 1995; Ramos et al., 1998). A memória EEPROM de $2 \mathrm{~Kb}$ (pode atingir $8 \mathrm{~Kb}$ no sistema Tiny Tag ${ }^{\circledR}$ ) possibilita o armazenamento de 1800 dados, com intervalos de registo pré-programados usando o software OTLM 1.41®. Trata-se de um sistema de aquisição de 8 bits que regista a temperatura através de um termistor NTC100, com funcionamento entre $-35^{\circ} \mathrm{C}$ e $70^{\circ} \mathrm{C}$, com uma precisão máxima de $0,35^{\circ} \mathrm{C}$. O sistema é alimentado por uma bateria de iões de lítio de 3,6v 1/2 AA, de muito bom rendimento a baixa temperatura (RAMOs, 1995), e que possibilita o funcionamento contínuo do registador ao longo de períodos superiores a 1 ano (segundo o fabricante, este pode chegar mesmo a 4 anos).

A recolha de dados e programação do sistema faz-se através de um computador portátil com um cabo RS232 ligado à porta de série. O software OTLM $1.41 \circledR$ permite uma fácil transferência dos dados, a sua visualização, e exportação para qualquer folha de cálculo corrente (fig. 1).

\section{Extracção do termistor}

Quando adquirido, o sistema Tiny Talk II ${ }^{\circledR}$ é uma peça única e compacta, onde se encontra embutido o termistor NTC100. A utilização do sistema com o termistor embutido deverá ser feita com especiais cuidados, pois a temperatura do próprio aparelho pode facilmente influenciar a temperatura do sensor. Além disso, a sensibilidade deste às variações rápidas de temperatura fica 


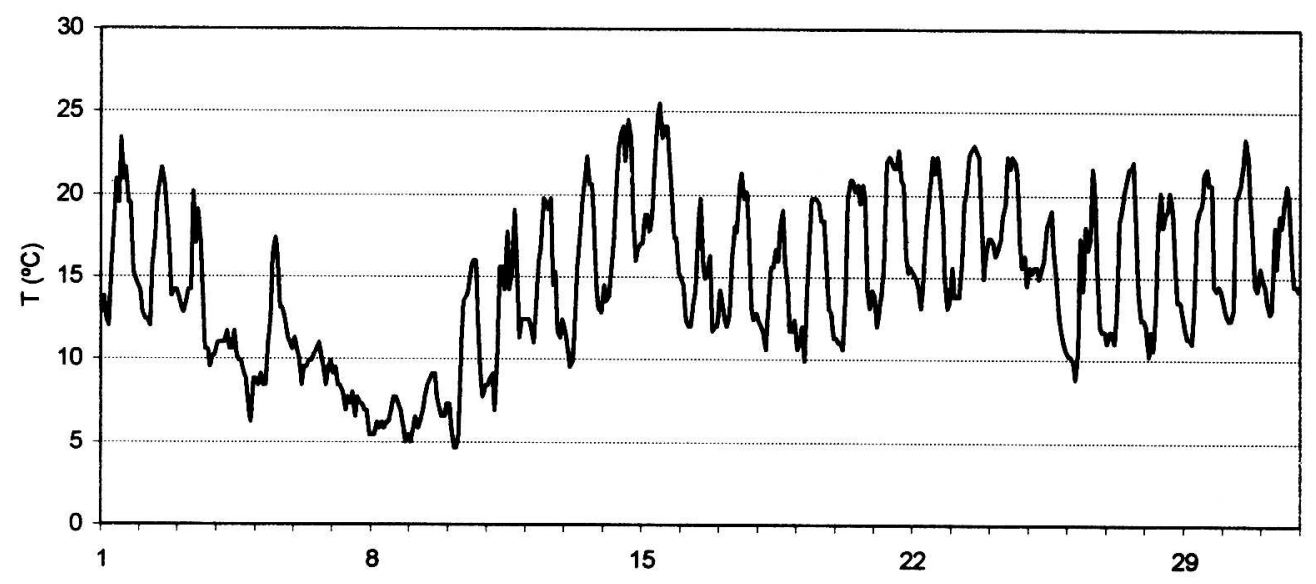

Fig. 1 - Evolução da temperatura do ar no Cântaro Gordo em Agosto de 1999.

Fig.1 - Temperatures in Cântaro Gordo Peak in August 1999 measured using the air temperature monitoring device (measurement interval $=90 \mathrm{~min})$.

certamente limitada pelo contacto com o suporte plástico do sistema de aquisição.

Para minimizar os efeitos referidos, para proteger melhor o sistema das condições ambientais e para lhe dar uma melhor protecção mecânica, extraímos o termistor de toda a instrumentação que desenvolvemos. No nosso entender, este procedimento é fundamental para a medição correcta das temperaturas do ar e do solo embora, neste último caso, em intervalos de medição longos, se possa utilizar o sensor sem o extrair do corpo do sistema de aquisição, devido à inércia térmica do solo. Trata-se de uma operação simples de efectuar, mas que requer alguns cuidados especiais.

O sistema de aquisição de dados deve ser fixado a um torno de mesa, e o termistor deve ser retirado com o auxílio de uma pinça (que segura o termistor) e de um ferro de soldar de ponta fina, que deverá ser usado para fundir a solda, que fixa o termistor, do lado oposto da placa. Dever-se-á ter o cuidado de não aquecer demasiado a solda, pois uma temperatura muito elevada danifica o termistor.

Uma vez extraído, o termistor deverá ser fixado a um fio eléctrico que funciona como ligação ao sistema de aquisição de dados, e que permite que o sensor se mantenha isolado, e em contacto directo com o ar. $\mathrm{O}$ extremo oposto do fio eléctrico deverá ser soldado ao sistema de aquisição, ocupando os orifícios de onde foi extraído o termistor.

Este procedimento foi usado para todos os instrumentos desenvolvidos, tendo sido posteriormente colocado o sensor de diferentes formas, em função do objectivos a que se destinava como descrevemos a seguir. 


\section{Instrumentação para a temperatura do ar}

Algumas das características dos instrumentação para a monitorização da temperatura do ar, que considerámos importantes, foram a leveza, o preço acessível e a facilidade de transporte, pois em alguns locais da serra da Estrela, a instalação das estações implica escalar algumas vertentes. Assim, além do sistema de aquisição de dados Tiny Talk II ${ }^{\circledR}$, a estação inclui uma estrutura de suporte construída à base de tubos de PVC, que possuem uma resistência aceitável, são baratos e bastante leves. O preço é um factor importante, pois os instrumentos são deixados no campo em áreas não vedadas e estão sujeitos a acções de vandalismo que, infelizmente, são frequentes. A estação é constituída por um pé de suporte, por uma cabeça amovível, que protege o sensor da radiação solar directa e pela unidade de registo devidamente acondicionada (fig. 2 ). Os materiais utilizados são de fácil aquisição no mercado.

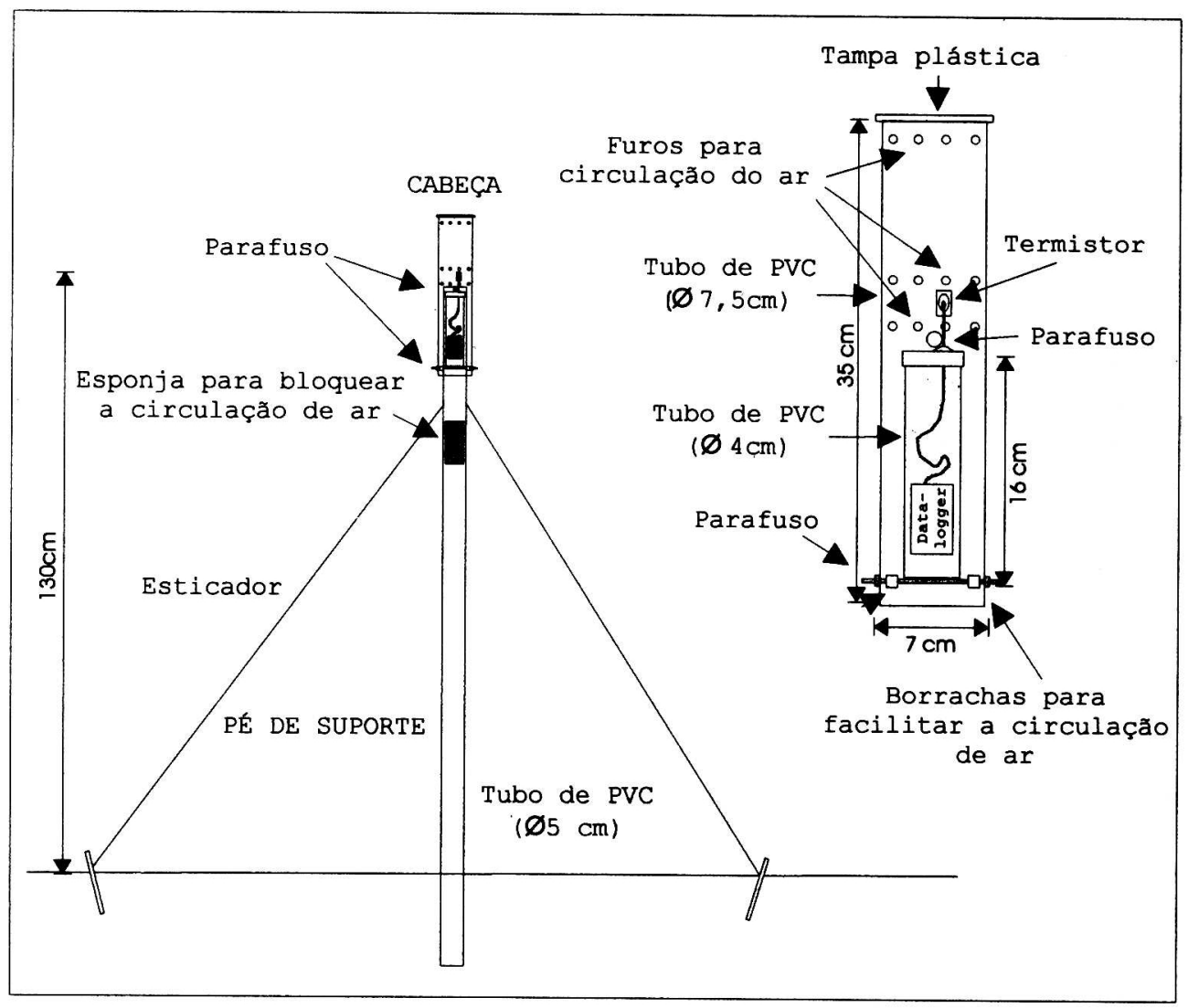

Fig. 2 - Características da estação para monitorização da temperatura do ar.

Fig.2 - Technical characteristics of the air temperature monitoring apparatus. 
O pé de suporte é constituído por um tubo de PVC de $5 \mathrm{~cm}$ de diâmetro com $150 \mathrm{~cm}$ de comprimento, que constitui uma medida padrão facilmente encontrada no mercado. O tubo é pintado de branco, e cerca de $20 \mathrm{~cm}$ abaixo do sensor é inserida uma esponja, de modo a minimizar a convecção no interior do tubo, associada às diferenças de temperatura nele verificadas. Para ancorar o tubo, enterra-se este no solo até cerca de $20 \mathrm{~cm}$ de profundidade, e utilizam-se 3 ou 4 esticadores para lhe dar maior resistência ao vento. $O$ sensor, que se colocará no extremo do tubo, fica então a cerca de $130 \mathrm{~cm}$ do solo, altura suficiente para que a influência deste seja minimizada.

A cabeça da estação é constituída por um tubo de PVC de $7 \mathrm{~cm}$ de diâmetro e $35 \mathrm{~cm}$ de comprimento, coberto no extremo superior por um tampão plástico, colado com Araldite ${ }^{\circledR}$. Toda a cabeça é pintada de branco. Três conjuntos de furos, alinhados com cerca de $4 \mathrm{~mm}$ de diâmetro, possibilitam uma melhor circulação do ar na cabeça, a qual é facilitada também pelo espaçamento existente no contacto entre a base da cabeça e o pé de suporte (fig.2).

$\mathrm{O}$ sistema de aquisição de dados fica protegido dentro de um tubo de PVC de $4 \mathrm{~cm}$ de diâmetro e cerca de $16 \mathrm{~cm}$ de comprimento (fig. 2). O tubo deverá ser tapado num dos extremos com um tampão, o qual deverá ter um pequeno furo por onde passa o termistor. É fundamental que todas as juntas, susceptíveis de deixar passar humidade, sejam totalmente isoladas com Araldite ${ }^{\circledR}$. A ligação entre o termistor e o fio eléctrico deve estar bem isolada, se possível com manga termoretráctil, e também com Araldite ${ }^{\circledR}$. O termistor é colado a um pequeno pedaço de chapa metálica de boa difusividade, que facilita o contacto com o ar, e a colocação da cola isolante referida acima. No interior do tubo fica colocado o sistema de aquisição de dados, que deve ter uma ligação ao termistor suficientemente longa, para que seja possível extrair o sistema pela extremidade oposta do tubo, de modo a poder proceder-se à recolha dos dados. Essa extremidade do tubo, deve estar isolada por um outro tampão, com as margens cortadas (de modo a poder ser encaixado no pé da estação), que será retirado, para a recolha de dados. Para impedir a entrada de humidade por este extremo, usamos cinta de canalização no interior do tubo, e fita isoladora de boa qualidade na parte externa.

Para proceder à montagem da estação, é ainda necessário efectuar os furos que permitem fixar a cabeça ao pé de apoio. Estes devem estar localizados no sector inferior e intermédio da cabeça, com um espaçamento suficiente que permita a inserção do tubo com o sistema de aquisição de dados. Deverão ser feitos em direcções ortogonais, para maximizar a resistência mecânica e estar alinhados com os furos respectivos a efectuar no pé de apoio. A fixação faz-se através de um parafuso que atravessa a cabeça e é fixo no lado exterior com porcas. No parafuso inferior, utilizamos anilhas de borracha com $5 \mathrm{~mm}$ de espessura, para manter um afastamento simétrico entre a cabeça e o pé de suporte, e possibilitar uma circulação mais homogénea do ar.

A montagem dos vários componentes da estação é feita aquando da instalação no campo. Primeiro, procedemos à instalação do pé de suporte e respec- 
tivos esticadores; segue-se a inserção do sistema de aquisição de dados, que fica encaixado no sector superior do pé de suporte e, finalmente, instala-se a cabeça amovível (fig. 3).

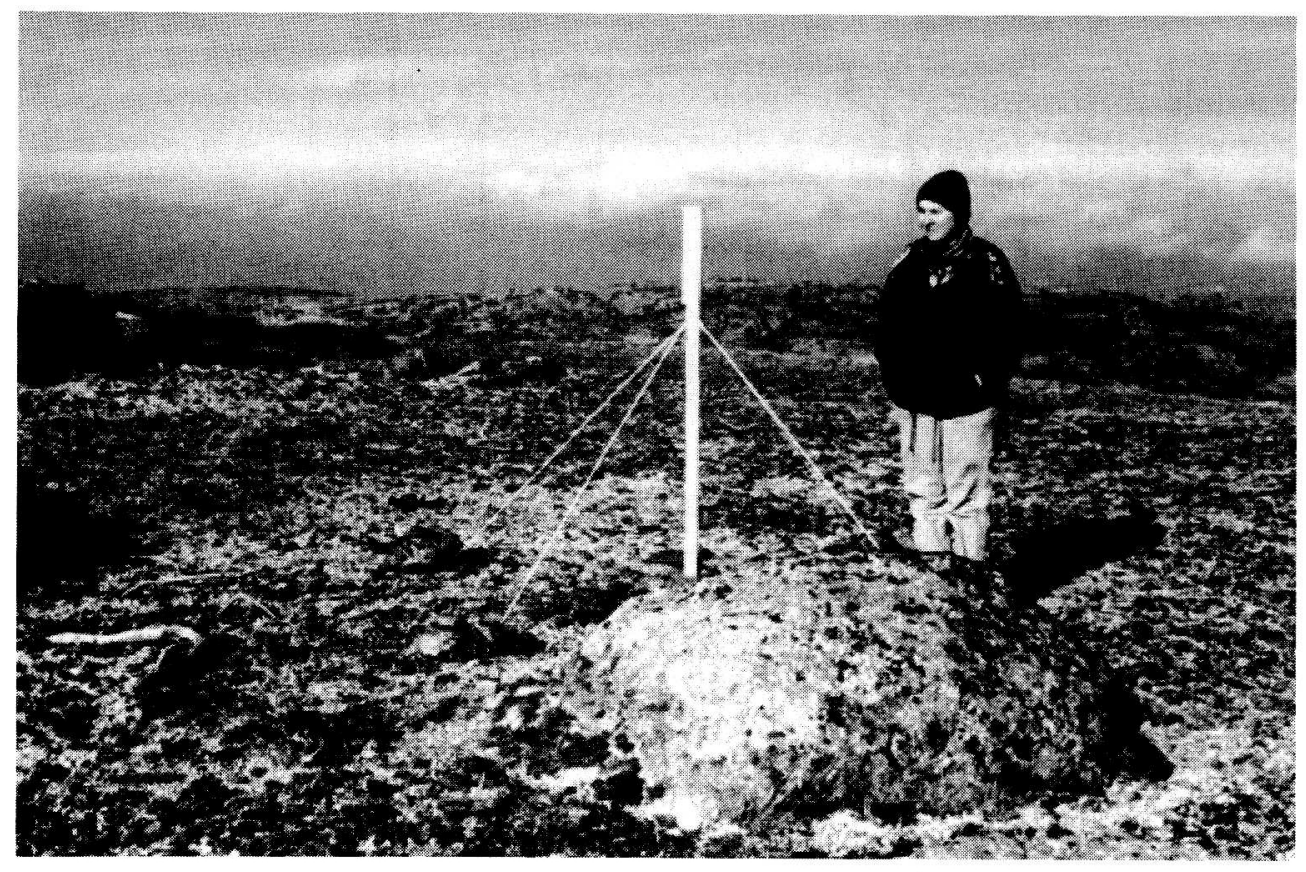

Fig. 3 - Estação para monitorização da temperatura do ar em funcionamento.

Fig. 3 - Air temperature monitoring apparatus.

\section{Instrumentação para a temperatura do solo}

O sistema desenvolvido permite a monitorização de temperaturas do solo a várias profundidades através da utilização de 4 termistores, ligados a sistemas de aquisição de dados independentes. Utilizam-se, por isso, 4 sistemas Tiny Talk II ${ }^{\circledR}$ instalados numa mesma caixa estanque, norma IP68 (fig. 4). Os registadores ficam encerrados na caixa, e desta saem apenas os 4 fios eléctricos que fazem a ligação aos termistores localizados no exterior. Os fios devem ser muito finos, de secção circular (podem ser separados posteriormente), e não terem um preenchimento de algodão, o qual funciona como um eficaz meio de transporte de humidade para o interior da caixa estanque, no caso de alguma pequena deficiência de isolamento. A passagem dos fios eléctricos pela parede da caixa deve ser feita através de furos com um diâmetro próximo ao dos fios e, se possível, mesmo ligeiramente inferior, pois a elasticidade destes permite a sua passagem. Estas passagens são isoladas com Araldite ${ }^{\circledR}$ para impermeabilização. 

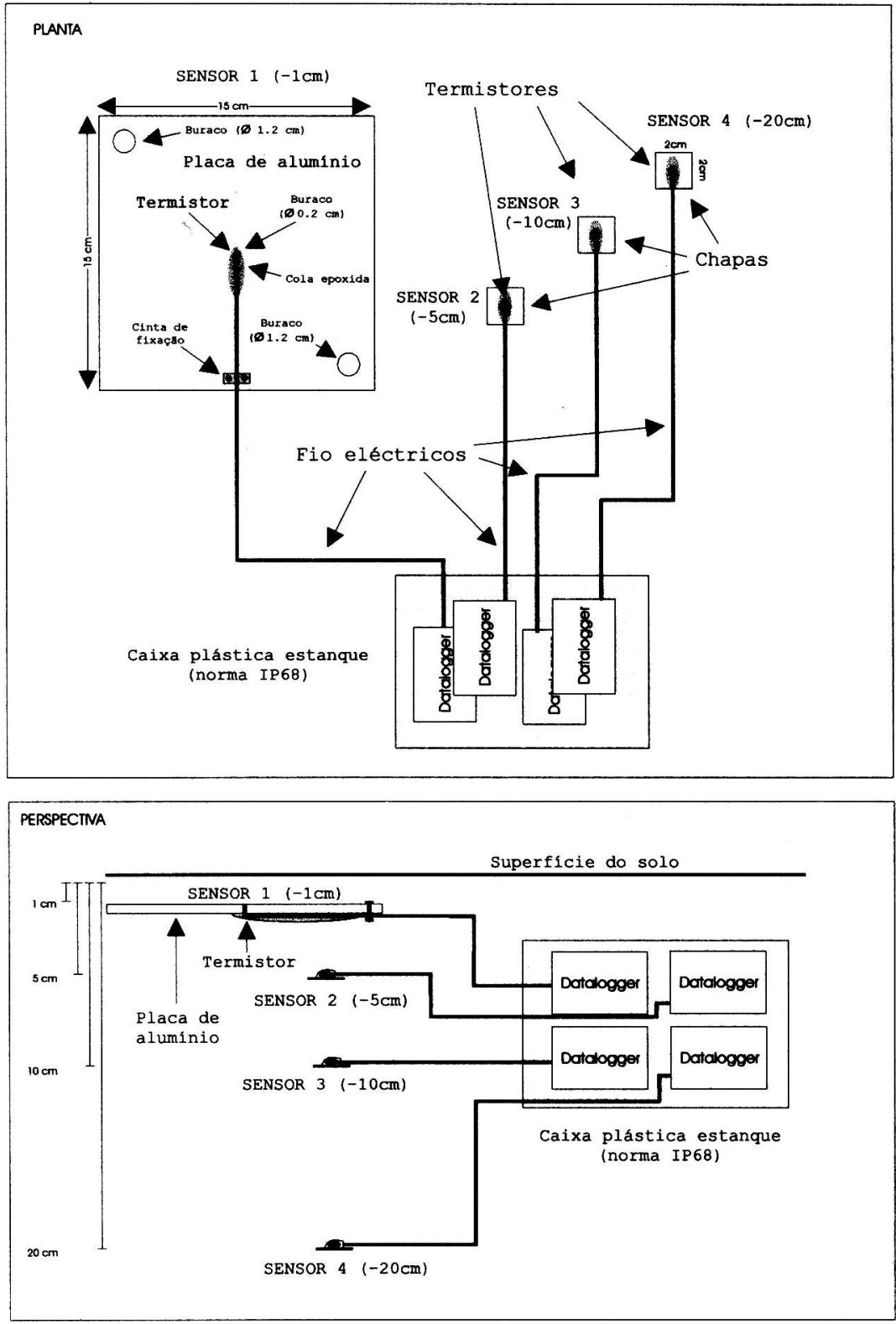

Fig. 4 - Características da estação para monitorização da temperatura do solo. Fig. 4 - Technical characteristics of the soil temperature monitoring apparatus.

Um aspecto importante para a medição da temperatura do solo, é estabelecer um contacto homogéneo com o sensor, de modo a minimizar os erros de leitura, resultantes da porosidade, e diferentes características térmicas dos 
componentes do solo. Este facto é especialmente importante quando se medem as temperaturas próximo da superfície, onde a porosidade é maior, e a influência da radiação solar mais efectiva. Os sensores foram por isso colados em chapas metálicas de boa difusividade, que permitem um melhor contacto com o solo, e obter assim, uma temperatura ponderada pelo efeito dos vários elementos que com ela contactam. O sensor que se localiza próximo da superfície, a cerca de $1 \mathrm{~cm}$ de profundidade, foi embutido numa placa de alumínio de $15 \times 15 \mathrm{~cm}$ e $0,5 \mathrm{~cm}$ de espessura (RAmos, 1995; Ramos et al., 1998). Os termistores, que se localizam a profundidades maiores, foram colados em chapas metálicas finas com $2 \times 2 \mathrm{~cm}$.

Para a instalação da instrumentação, a caixa deve ficar enterrada ao lado dos sensores, a uma profundidade intermédia, entre o sensor mais superficial e o mais profundo. Minimiza-se assim o efeito de 'ponte térmica', causado pela condução através dos cabos, e que pode influenciar a temperatura dos sensores.

\section{Instrumentação para a temperatura da rocha}

A instrumentação de monitorização de temperatura da rocha é muito semelhante à do solo. É usada a mesma caixa para protecção dos sistemas de aquisição de dados, e o processo de passagem dos fios eléctricos para o exterior é o mesmo. A diferença principal reside no facto de o objectivo ser medir as temperaturas num meio sólido, e as diferenças centram-se na instalação dos sensores, que ficam embutidos na rocha a diferentes profundidades (fig.5).
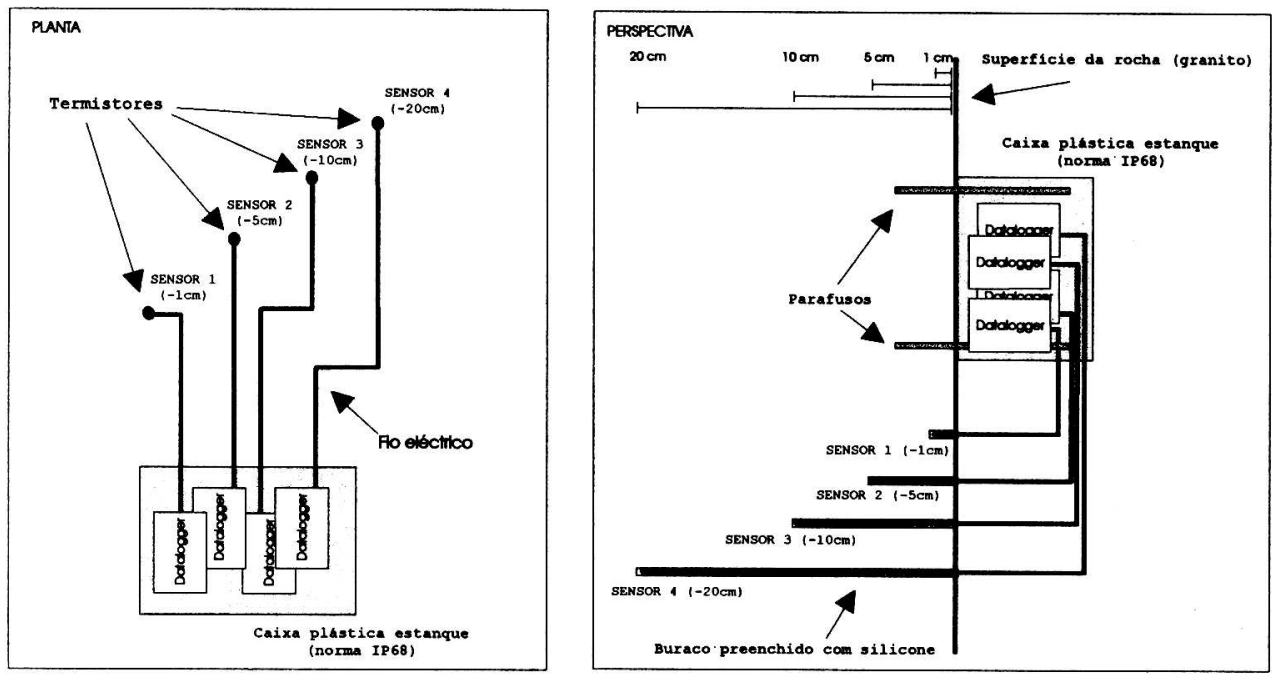

Fig. 5 - Características da estação para monitorização da temperatura da rocha Fig. 5 -Technical characteristics of the rock temperature monitoring apparatus. 
Os termistores são instalados na ponta dos fios eléctricos, que os ligam ao sistema de aquisição de dados, e devem ser devidamente isolados para impedirem a migração de humidade do exterior para o interior da rocha. A utilização de uma manga termoretráctil torna a secção do fio mais regular, e deverá ajudar a resolver o problema. Mas os principais cuidados devem ser tomados durante a instalação do sistema na superfície rochosa a estudar. Os furos devem ser efectuados com uma broca fina e devem ser de espessura regular. Cada sensor fica alojado num furo distinto, e encostado cuidadosamente ao fundo do buraco, o qual é preenchido com cola de silicone (MATSUOKA, 1990), que funciona como isolante minimizando a condução térmica desde o exterior. A caixa deverá ser colocada próximo dos sensores, mas numa posição que não influencie os registos obtidos (por efeito de sombra, ou por acumulação preferencial de neve).

\section{AGRADECIMENTOS}

Os autores agradecem ao Sr. José Maria Saraiva pela incansável ajuda prestada nos trabalhos de campo, que em muito contribuiu para a bem sucedida instalação das estações de medida, ao Dr. Mário Neves e aos Srs. Joaquim Neves, António Eanes e Pedro Mora pela ajuda na construção e instalação das estações, ao Dr. Henrique Andrade pelos conselhos relativos ao desenho das estações para a monitorização da temperatura do ar, ao Prof. Norikazu Matsuoka pelos comentários relativos à melhor forma de instalar os sensores na rocha e ao Dr. Adriano Ribolini pelas sugestões na escolha do sistema de aquisição de dados.

Esta investigação foi desenvolvida no âmbito do Projecto Processos geomorfológicos e biofísicos e unidades de paisagem em ambiente de montanha mediterrânea. Aplicação à serra da Estrela (ESTRELA), financiado pela Fundação para a Ciência e a Tecnologia (Praxis/C/CTE/11153/1998).

\section{BIBLIOGRAFIA}

Alcoforado, M. J.; Andrade, E.; Neves, M.; Vieira, G. (1993) - Contribuição para o estudo dos climas locais da Arrábida no Inverno, Finisterra, XXVII (55-56): 215-228.

Matsuoka, N. (1990) - The rate of bedrock weathering by frost action: field measurements and a predictive model, Earth Surface Processes and Landforms, 15: 73-90

Ramos, M. (1995) - Automatic device to measure the active permafrost layer near the Spanish Antarctic Station, Terra Antarctica, 2 (1): 61-63.

Ramos, M.; Gómez Ortiz, A.; Salvador Franch, F., Schulte, L. (1998) - Evolución térmica de la capa activa en la estación geomorfológica de la planicie La Feixa-La Màniga, 2.150 m (macizo de Calmquerdós. Pirineo Oriental), in A. Gómez Ortiz, F. Salvador Franch, L. Schulte and A. García Navarro, eds., Procesos biofísicos actuales en medios fríos. Estudios recientes, Publicaciones Universitat de Barcelona: 73-97.

Thorn, C. E.; Schlyter, J. P. L.; Darmody, R.; Dixon, J. (1999) - Statistical relationships between daily and monthly air and shallow-ground temperatures in Kärkevagge, Swedish Lapland, Permafrost and Periglacial Processes, 10: 317-330. 